APS/123-QED

\title{
Atomistic treatment of depolarizing energy and field in ferroelectric nanostructures
}

\author{
I. Ponomareva, I. I. Naumov, I. Kornev, Huaxiang Fu and L. Bellaiche \\ Department of Physics, University of Arkansas, \\ Fayetteville, Arkansas 72701, USA
}

(Dated: September 13, 2018)

\begin{abstract}
An atomistic approach allowing an accurate and efficient treatment of depolarizing energy and field in any low-dimensional ferroelectric structure is developed. Application of this approach demonstrates the limits of the widely used continuum model (even) for simple test cases. Moreover, implementation of this approach within a first-principles-based model reveals an unusual phase transition - from a state exhibiting a spontaneous polarization to a phase associated with a toroid moment of polarization - in a ferroelectric nanodot for a critical value of the depolarizing field.
\end{abstract}

PACS numbers: 77.22.Ej, 77.80.Bh, 77.84.Dy 
Ferroelectric nanostructures (FEN) are of increasing technological and fundamental interest because of the need in miniaturization of devices, as well as, the appearance of new phenomena (see, e.g., Ref. [1, 2, 3, 4, 5, 6] and references therein). Unscreened polarizationinduced charges at the surfaces of FEN generate a depolarizing field that is responsible for striking properties. Examples are the existence of a critical thickness below which no ferroelectricity can appear [3], and the observation and prediction of laminar stripe nanodomains [2, 4] as well as the formation of polarization vortex [5, 6]. Interestingly, and despite its huge importance, we are not aware of any model being able to exactly calculate the depolarizing field and energy in any low-dimensional ferroelectric. For instance, the widely used continuum model (1) neglects the atomistic nature of materials, (2) is technically applicable only in the limit of large enough systems and (3) can not predict the depolarizing energy/field in the realistic cases of inhomogeneously polarized samples.

In this Letter we i) demonstrate that it is possible to derive a scheme allowing the exact atomistic computation of the depolarizing energy and field in any low-dimensional FEN; ii) use this scheme to check the accuracy of the continuum model for some simple test cases; iii) report an unusual phase transition between two different kinds of order parameter in a ferroelectric nanodot that is driven by the depolarizing field.

To calculate the depolarizing energy in low-dimensional ferroelectrics, one first needs to realize that a system under perfect open-circuit (OC) electrical boundary conditions exhibits a maximum depolarizing field (if the polarization lies along a non-periodic direction), while ideal short-circuit (SC) electrical boundary conditions leads to a complete screening of charges at the FEN surfaces that fully annihilates any depolarizing field. As a result, the depolarizing energy and field experienced by the FEN should involve a difference between the dipole-dipole interactions associated with these two extreme electrical boundary conditions. We shall write energy of the dipole-dipole interaction in any system in the form

$$
\mathcal{E}_{d i p}^{(D)}=\frac{1}{2 V} \sum_{\alpha \beta, i j} Q_{\alpha \beta, i j}^{(S, D)} p_{\alpha}\left(\mathbf{r}_{i}\right) p_{\beta}\left(\mathbf{r}_{j}\right),
$$

where $D=3,2,1$ stands for a system periodic in 3,2 and 1 directions, respectively; $D=0$ corresponds to non-periodic systems and the sum runs over the atomic sites $i$ and $j$ that differ from each other and belong to a supercell (to be denoted by $S$ ) mimicking the system. Such a supercell is infinitely repeated along the periodic directions, if any. For instance, 
thin films are modeled by supercells that are repeated in two dimensions while the direction associated with the growth direction of the film is non-periodic. For dots, the supercell is not repeated. $\mathrm{V}$ is the volume of the supercell, $\boldsymbol{p}\left(\boldsymbol{r}_{i}\right)$ the dipole moment at the site $i$, $\alpha=x, y, z$ denotes the Cartesian components. The quantity $Q^{(S, D)}$ depends on both the chosen supercell $(S)$ and the periodicity of the system $(D)$.

It is straightforward to prove that the elements of the $\mathrm{Q}$ matrix for systems periodic in three 7], two $(x$ - and $y)$, one $(z)$ directions [20] and non-periodic systems are given by:

- $Q_{\alpha \beta, i j}^{(S, 3)}=\frac{4 \pi}{V} \sum_{\mathbf{G} \neq 0} \frac{1}{G^{2}} \exp \left(-\frac{G^{2}}{4 \lambda^{2}}\right) G_{\alpha} G_{\beta} \cos \left(\mathbf{G r}_{i j}\right)-\frac{4 \lambda^{3} \delta_{\alpha \beta} \delta_{i j}}{3 \sqrt{\pi}}$,

- $Q_{\alpha \beta, i j}^{(S, 2)}=\frac{2 \pi}{A} \sum_{\mathbf{G}}\left\{G \cos \left(\mathbf{G} \cdot \boldsymbol{\rho}_{i j}\right)\left[\frac{1}{\sqrt{4 \pi}} \Gamma\left(-\frac{1}{2}, \frac{G^{2}}{4 \lambda^{2}}\right) \delta_{\alpha z} \delta_{\beta z}+\frac{1}{G^{2}} \operatorname{erfc}\left(\frac{G}{2 \lambda}\right) G_{\alpha} G_{\beta}\right]\right.$

$\left.+G \exp \left(-G\left|z_{i j}\right|\right)\left[\left(\frac{G_{\alpha} G_{\beta}}{G^{2}}-\delta_{\alpha z} \delta_{\beta z}\right) \cos \left(\mathbf{G} \cdot \boldsymbol{\rho}_{i j}\right)-\frac{G_{\alpha} \delta_{\beta z}}{G} \sin \left(\mathbf{G} \cdot \boldsymbol{\rho}_{i j}\right) \frac{z_{i j}}{\left|z_{i j}\right|}\right]\right\}-\frac{4 \lambda^{3} \delta_{\alpha \beta} \delta_{i j}}{3 \sqrt{\pi}}$,

- $Q_{\alpha \beta, i j}^{(S, 1)}=\frac{2}{a} \sum_{\mathbf{G}} G^{2} \cos \left(\mathbf{G} \cdot \mathbf{z}_{i j}\right)\left\{K_{0}\left(G \rho_{i j}\right) \delta_{\alpha z} \delta_{\beta z}+\frac{\delta_{\alpha x} \delta_{\beta x}+\delta_{\alpha y} \delta_{\beta y}}{G \rho_{i j}} K_{1}\left(G \rho_{i j}\right)\right.$

$\left.-\frac{1}{\rho_{i j}^{2}} K_{2}\left(G \rho_{i j}\right) \rho_{\alpha, i j} \rho_{\beta, i j}\right\}-\frac{2}{a} \sum_{\mathbf{G}} G \sin \left(\mathbf{G} \cdot \mathbf{z}_{i j}\right) K_{1}\left(G \rho_{i j}\right) \rho_{i j}^{-1} \times G_{\alpha} \rho_{\beta, i j}+$

$+\frac{1}{a^{3}}\left(\delta_{\alpha x} \delta_{\beta x}+\delta_{\alpha y} \delta_{\beta y}-2 \delta_{\alpha z} \delta_{\beta z}\right) \sum_{n=-\infty}^{\infty}\left|n+\frac{z_{i j}}{a}\right|^{-3}$,

- $Q_{\alpha \beta, i j}^{(S, 0)}=\frac{\delta_{\alpha, \beta}}{r_{i j}^{3}}-\frac{3 r_{\alpha, i j} r_{\beta, i j}}{r_{i j}^{5}}$

where $\mathbf{G}$ are the reciprocal lattice vectors associated with the $S$ supercell, $\lambda$ is the Ewald parameter [8] (which is assumed to be large enough), $\delta_{i j}$ is the Kronicker symbol, $A$ is the supercell area, $\boldsymbol{\rho}_{i j}$ and $z_{i j}$ are the projections of $\boldsymbol{r}_{i j}$ (vector connecting atomic sites $i$ and $j$ ) on the $\{x, y\}$ plane and $z$-axis, respectively. $\Gamma$ is the incomplete Gamma function and $\operatorname{erf} c$ is the complementary error function, $a$ is the supercell length in $z$-direction; $K_{n}$ are the modified Bessel's functions. (Note, that contributions from $z_{i j}=0$ in $Q_{\alpha \beta, i j}^{(S, 2)}$ and contributions from $\rho_{i j}=0$ in $Q_{\alpha \beta, i j}^{(S, 1)}$ should be excluded, and that the prime in the right side of $Q_{\alpha \beta, i j}^{(S, 1)}$ indicates that the term $n=0$ has to be excluded when $i=j$.

The dipolar interactions described by equations (11) and (2) correspond to ideal OC conditions since no charge screening is taken into account in their derivation. The next question to be addressed is what is the dipole-dipole energy in FEN under perfect $S C$ conditions. Such energy is simply the one described by $D=3$ Q-matrix since infinitely extended (i.e, bulk) systems do not experience any macroscopic depolarizing field[9]. One can also be 
convinced in the correctness of the above statement by applying the so-called image method that produces polarization pattern identical to the one in the bulk from the polarization pattern in the nanostructure.

The $\mathcal{E}_{d e p}^{(D)}$ (maximum) depolarizing energy per volume in any FEN can now easily be calculated as the difference in dipole-dipole energies between perfect $\mathrm{OC}$ and $\mathrm{SC}$ conditions, that is:

$$
\mathcal{E}_{d e p}^{(D)}=\mathcal{E}_{d i p}^{(D)}-\mathcal{E}_{d i p}^{(3)}=\frac{1}{2 V} \sum_{\alpha \beta, i j}\left[Q_{\alpha \beta, i j}^{(S, D)}-Q_{\alpha \beta, i j}^{(S, 3)}\right] p_{\alpha}\left(\mathbf{r}_{i}\right) p_{\beta}\left(\mathbf{r}_{j}\right)
$$

where the sum over $i$ and $j$ run over the sites of the chosen supercell of the FEN, and where $Q_{\alpha \beta, i j}^{(S, D)}$ are given by Eq.(2). Equation (3) is, to the best of our knowledge, the first proposed form allowing an atomistic and exact computation of depolarizing energy in any FEN with any dipole distribution. (Note that such form can also be applied to calculate demagnetization energy in low-dimensional magnetic systems).

We first apply our approach to compute $\mathcal{E}_{\text {dep }}^{(D)}$ in some test cases. Here, we limit ourselves to systems adopting a simple cubic structure in which each atomic site has a local and equal-in-magnitude dipole moment. Note, that all results to be reported here do not depend on the size used for the periodic direction(s) of the $S$ supercell.

Homogeneous dipole distribution: let's first investigate FEN exhibiting the same local dipole $\mathbf{p}$ at any atomic site. We shall present our results in the form of $\mathcal{E}_{\text {dep }}^{(D)}=\gamma \mathcal{E}_{\text {dep }}^{(D, c o n t)}$, where $\mathcal{E}_{\text {dep }}^{(D)}$ is obtained from Eq.(33), while $\mathcal{E}_{\text {dep }}^{(D, c o n t)}$ is the depolarizing energy predicted by the continuum model. $\gamma$ is thus a "correcting" coefficient that provides a measure of the continuum approach accuracy.

For (001) ultra-thin films homogeneously polarized along the out-of-plane $(z)$ direction, the continuum model predicts that $\mathcal{E}_{\text {dep }}^{(2, c o n t)}=2 \pi P^{2}$, independently of the film thickness, where $P$ is the polarization. On the other hand, the use of Eq (3) results in $\gamma=1.017,1.010$, 1.007 and 1.006 for ultra-thin films of 3, 5, 7 and 9 layers, respectively. In other words, our atomistic approach reveals that the depolarizing energy is slightly larger than the one predicted by the continuum model and increases as the number of film layers decreases. Such findings are consistent with those of Refs. 10, 11, 12]. To understand them, we rewrite $\mathrm{Eq}$ (3) in the case of a homogeneous dipole pattern as follows:

$$
\mathcal{E}_{d e p}^{(D)}=\frac{p_{\alpha} p_{\beta}}{2 V} \sum_{\alpha \beta, j} F_{\alpha \beta}^{(D)}(j), \text { with } F_{\alpha \beta}^{(D)}(j)=\sum_{i}\left[Q_{\alpha \beta, i j}^{(S, D)}-Q_{\alpha \beta, i j}^{(S, 3)}\right]
$$


Fig 11a shows the "depolarizing" factors $<F_{z z}^{(D=2)}(l)>$ averaged over all the $j$ sites belonging to a given (001) layer (that is indexed by $l$ ) for our films, as a function of a layer position inside the film. Comparison with its continuum predicted (and $l$-independent) value of $4 \pi$ is also given. Fig 19 clearly reveals that the deviation of the continuum model from our atomistic results is confined to the surface layers - as also found in Ref.[12] - and that this deviation is an underestimation. This explains why we numerically found that the $\mathcal{E}_{\text {dep }}^{(2)}$ increases with respect to the continuum prediction as the film becomes thinner, since the ratio of surface layers over total layers increases as the film thickness decreases. Fig 19 also shows that for the surface layers $\left\langle F_{x x}^{(2)}\right\rangle=\left\langle F_{y y}^{(2)}\right\rangle$ are negative - as discussed in Ref. 11] - and that $1+\left(<F_{x x}^{(2)}>-<F_{z z}^{(2)}>\right) / 4 \pi=-0.0393$, which is in perfect agreement with the calculation of the so-called surface anisotropy in Ref.[12].

We next consider different wires of square cross sections, that are periodic along the $z$-direction and homogeneously polarized along the $x$-axis. According to the continuum approach, such wires should have a depolarizing energy $\mathcal{E}_{\text {dep }}^{(1, \text { cont })}=\pi P^{2}$, independently of the wire thickness. We numerically found via Eq. (3) that $\gamma=1.017,1.010,1.007$ and 1.006 for wires of 3, 5, 7 and 9 shells, respectively (see inset of Fig 1p for definition of shells). Like in the films, the continuum model underestimates the depolarizing energy and this underestimation becomes larger in magnitude as the nanostructure shrinks in size. Fig 1b shows that the continuum model fails to reproduce the averaged depolarizing factor for the surface shell but exactly agrees with our atomistic results for all the inner shells.

We now turn our attention to a cubic dot homogeneously polarized along the z-direction. Unlike the previous two cases, our atomistic approach gives a depolarizing energy that is not only independent on the dot size but also exactly agrees with the continuum approach (that is, $\left.\mathcal{E}_{\text {dep }}^{(0)}=\mathcal{E}_{d e p}^{(0, c o n t)}=2 \pi P^{2} / 3\right)$. Such a surprising result is caused by the sum rule [1]] for depolarization factors ( that is, $F_{x x}+F_{y y}+F_{z z}$ is the constant given by the continuum model) that we numerically found to be valid in every layer (shell) of any system investigated so far (i.e. films, wires and dots). Moreover, cubic dots exhibit $x, y$ and $z$ directions that are symmetrically equivalent (which is not the case in wires and thin films). As a result, $<F_{x x}^{(D)}(l)>=<F_{y y}^{(D)}(l)>=<F_{z z}^{(D)}(l)>$ in dots. Because of the sum rule, each of these factors is equal to the continuum prediction of $4 \pi / 3$ for any shell and for any size, and the continuum model predicts the right depolarizing energy.

Inhomogeneous dipole distribution: let's now investigate 2D, 1D and 0D FEN exhibiting 
stripe domains with the dipoles assumed to be homogeneous inside each domain (with a $p$ magnitude) and perpendicular to a periodic direction, if any (see the inset of Fig (2). The period of stripe domains is denoted as $d$. The $\mathcal{E}_{\text {dep }}^{(D)}$ energy calculated from Eq (3) for a film having a thickness $L=10$ atomic layers is shown as a function of $d / L$ in Fig 2, along with depolarization energies calculated in the continuum approach for the two limiting cases $d<<L$ (i.e, $\left.\mathcal{E}_{\text {dep }}^{(2, \text { cont })}=1.7 P^{2} d / L[13, \underline{14}]\right)$ and $d>>L[15]$. One can see that these two limiting cases can reproduce rather well the energy derived from Eq (3) for $d / L<1$ and $d / L>2$, respectively. Furthermore we numerically found that our depolarization energy can be parametrized as following

$$
\mathcal{E}_{\text {dep }}^{(D)}=\left[c_{0}\left(1-c_{1} e^{-d / c_{2} L}\right)+\left(n \pi-c_{0}\right)\left(1-e^{-d / c_{3} L}\right)\right] P^{2}
$$

where $c_{0}=2.568, c_{1}=1.024, c_{2}=14.118, c_{3}=1.831, n=2$.

Fig 2 also reports our results for two cases for which we are not aware of any continuum predictions, namely stripe domains in infinite wires and cubic dots. Note that the stripe direction is along the wire $y$ periodic direction and that the finite size of 0D systems implies that $d / L$ has a maximum value of 0.5 in cubic dots. We present here results for a wire of 10 shells (10x10 atomic sites for cross section) and for a cubic dot of 10 shells (10x10x10 atomic sites) with $d$ ranging from 1 to 5 atomic layers. Two features seen in Fig 2 are particularly striking. First of all, the stripe domains have less depolarizing energy in a wire than in a thin film for the same $d / L$, with this difference becoming more pronounced as $d / L$ increases. The parameters of equation (5) for the wire are $c_{0}=2.208, c_{1}=1.000, c_{2}=1.196, c_{3}=7.398$ and $n=1$. Secondly, for the case of a cubic dot, the dependence of depolarizing energy on $d / L$ is linear and given by $\mathcal{E}_{d e p}^{(0)}=2.5 P^{2} d / L$.

We finally take advantage of our formalism to reveal, from realistic first-principles-based calculations, properties of a free standing $\mathrm{Pb}\left(Z r_{0.4} T i_{0.6}\right) O_{3}$ (PZT) cubic dot of $48 \AA$ lateral size for different electrical boundary conditions. The total energy of the system used in Monte Carlo simulations is:

$$
\mathcal{E}_{H e f f}\left(\mathbf{p}\left(\mathbf{r}_{i}\right), \mathbf{v}_{i}, \eta, \sigma_{i}\right)+\beta \sum_{i}<\mathbf{E}_{d e p}>\mathbf{p}\left(\mathbf{r}_{i}\right)
$$

where $\mathcal{E}_{\text {Heff }}$ is the (first-principles-derived Effective Hamiltonian) energy for PZT [16] which is dependent on the $\mathbf{p}\left(\mathbf{r}_{i}\right)$ local dipoles at site $i$ of the dot, the $\mathbf{v}_{i}$ inhomogeneous strain related variables, the $\eta$ homogeneous strain tensor, and on the $\sigma_{i}$ atomic configuration [16]. 
The dipole-dipole interactions in this $H_{\text {eff }}$ are given by the Q-matrix with $D=0$ of Eq2. The second term of equation (6) mimics a screening of the (maximum) depolarizing field, with the magnitude of this screening being controlled by the $\beta$ coefficient. $\beta=1$ and $\beta=0$ corresponds to ideal SC and OC electrical boundary conditions, respectively, while a value of $\beta$ in-between corresponds to more realistic electrical situation [3]. $<\mathbf{E}_{d e p}>=$ $-\left(1 / N \epsilon_{\infty}\right) \sum_{j}\left(\partial \mathcal{E}_{d e p}^{(D=0)} / \partial \mathbf{p}\left(r_{j}\right)\right)$ is the depolarizing field inside the dot, while $N$ and $\epsilon_{\infty}$ are the total number of sites of the dot and the dielectric constant of PZT, respectively. $\mathcal{E}_{d e p}^{(D=0)}$ is practically calculated from Eq. (3).

Fig $[3$ and b show the resulting macroscopic dipole moment and the macroscopic toroid moment of polarization (i.e., the supercell average of the cross product between position and dipole moment [6]), respectively, as a function of $\beta$. One can clearly see that for situations close to SC, the dot exhibits a macroscopic polarization, with a cross section of the local dipole pattern being given in the inset of Fig Ba. On the other hand a dot with electrical boundary conditions close to $\mathrm{OC}$ has a non-vanishing toroid moment [6] , with a cross-section of the corresponding dipole pattern being displayed in the inset of Fig 3 b. Moreover, Fig 3 clearly reveals that, at a critical value of the depolarization field, the system undergoes an unusual phase transition between a state characterized by one kind of order parameter (toroid moment of polarization) to a state associated with another kind of order parameter (polarization). In other words, no coexistence between these two order parameters occurs.

In summary, we have derived an atomistic, simple, general and efficient approach to calculate the depolarizing energy and field in any low-dimensional ferroelectric structure. The application of this method reveals - and explains - the limits of the continuum model, and also results in the discovery of an unusual phase transition in a ferroelectric dot for some critical value of the residual depolarizing field.

We thank P. Ghosez for discussion. This work is supported by NSF grants DMR-0404335 and DMR-9983678, by ONR grants D 00014-01-1-0365, D 00014-04-1-0413 and D 00014-011-0600 and by DOE grant DE-FG02-05ER46188.

[1] J. F. Scott and C. A. P. de Araujo, Science 246, 1400 (1989).

[2] D. Fong, G. Stephenson, S. Streiffer, J. Eastman, O. Auciello, P. Fuoss, and C. Thompson, 
Science 304, 1650 (2004).

[3] J. Junquera and P. Ghosez, Nature 422, 506 (2004).

[4] I. Kornev, H. Fu, and L. Bellaiche, Phys. Rev. Lett. 93, 196104 (2004).

[5] H. Fu and L. Bellaiche, Phys. Rev. Lett. 91, 257601 (2003).

[6] I. I. Naumov, L. Bellaiche, and H. Fu, Nature 432, 737 (2004).

[7] W. Zhong, D. Vanderbilt, and K. Rabe, Phys. Rev. B 52, 6301 (1995).

[8] C. Kittel, Introduction to Solid State Physics (John Wiley and Sons, Inc., 1996), pp. 386-388, seventh ed.

[9] B. Meyer and D. Vanderbilt, Phys. Rev. B 63, 205426 (2001).

[10] P. J. Jensen, Ann. Physik 6, 317 (1997).

[11] E. Vedemenko, H.P.Oepen, and J.Kirschner, J. Magnetism and Magn. Materials 256, 237 (2003).

[12] H. Draaisma and W. de Jonge, J. Appl. Phys. 64, 3610 (1988).

[13] C. Kittel, Phys. Rev. 70, 965 (1946).

[14] T. Mitsui and H. Furuichi, Phys. Rev. 90, 193 (1953).

[15] B. Kaplan and G. Gehring, J. Magn. Magn. Mater. 128, 111 (1993).

[16] L. Bellaiche, A. Garcia, and D. Vanderbilt, Phys. Rev. Lett 84, 5427 (2000).

[17] Y. J. Rhee, J. Halley, J. Hautman, and A. Rahman, Phys. Rev. B 40, 36 (1989).

[18] A. Brodka, Chem. Phys. Lett. 363, 604 (2002).

[19] A. Brodka and A. Grzybowski, J. Chem. Phys. 117, 8208 (2002).

[20] To calculate the elements of the Q-matrix we developed an original approach using periodic Green's function - satisfying Laplace equation and analytically determined. Details of this approach will be published elsewhere. Note that other approaches allowing the calculation of dipolar interactions can be found in Refs. 10, 17, 18, 19]. 

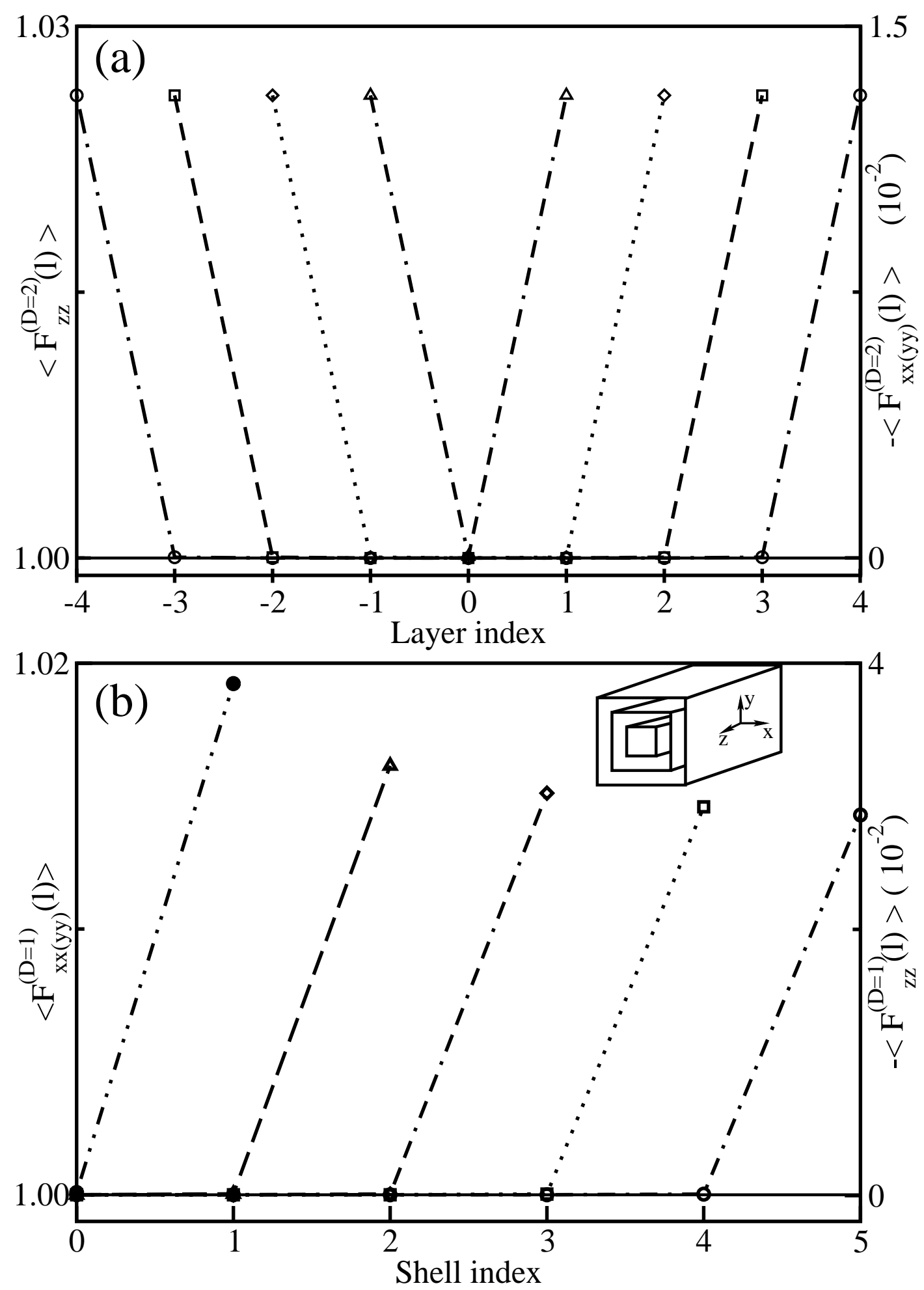

FIG. 1: Depolarizing factors $\left\langle F_{x x}^{(D)}(l)>,\left\langle F_{y y}^{(D)}(l)>\right.\right.$ and $\left\langle F_{z z}^{(D)}(l)>\right.$ obtained with our atomistic approach and normalized to $4 \pi$ (that is the prediction of $\mathrm{F}_{z z}$ in the continuum model) in the case of (001) films (part (a)) and $2 \pi$ (prediction of $\mathrm{F}_{x x}$ in the continuum model) in the case of wires periodic along the $z$-axis (part (b)) as a function of the layer or shell index $l$. The different shells of a wire are shown in the inset of part (b). The most inner layer or shell is indexed by 0 . 


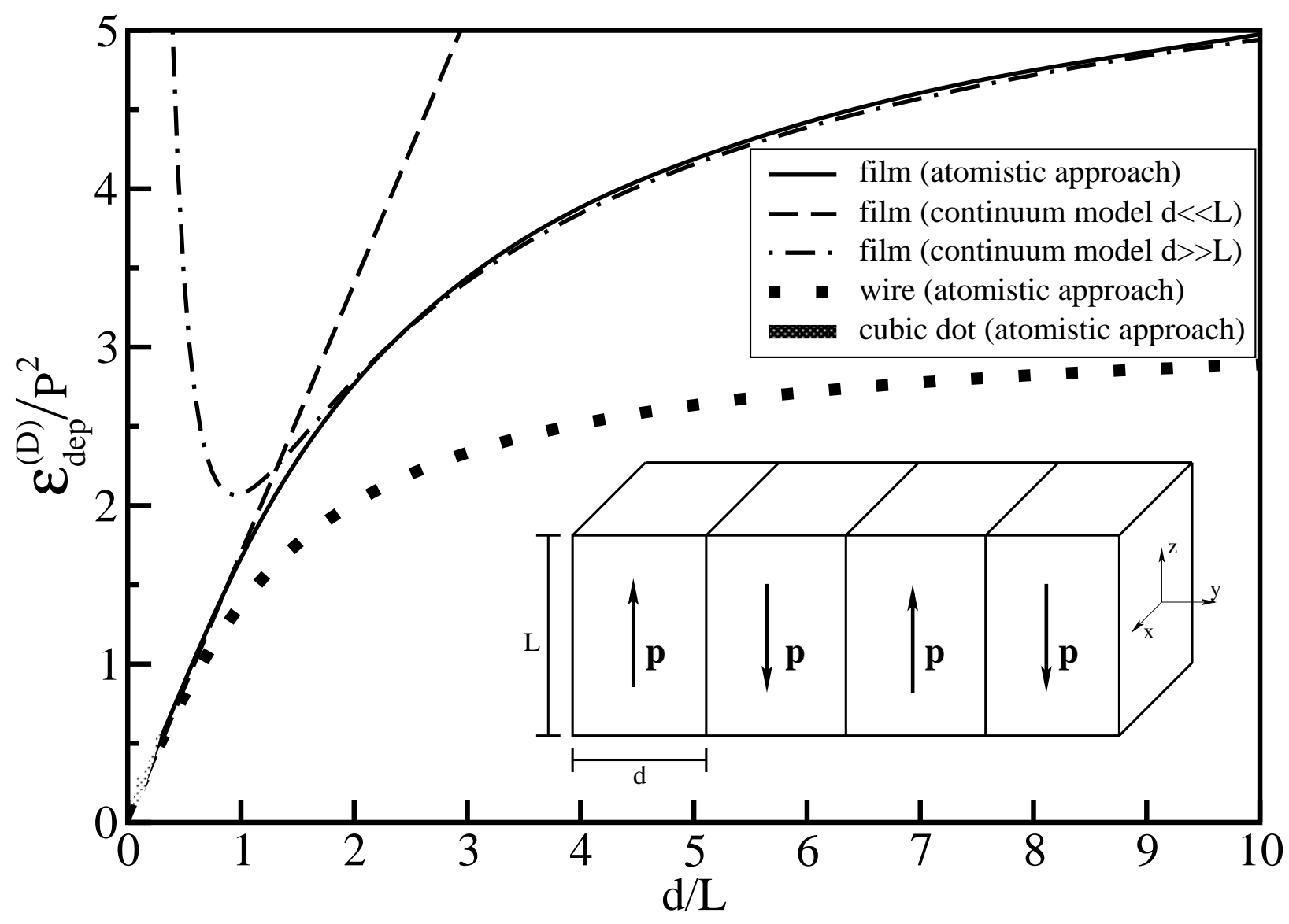

FIG. 2: Normalized depolarization energy as a function of $d / L$ for quasi $0 \mathrm{D}, 1 \mathrm{D}$ and $2 \mathrm{D}$ systems with stripe domains along with continuum model prediction for $d<<L[13]$ and $d>>$ [15] for $\mathrm{D}=2$ systems. The inset shows the schematic representation of the chosen polarization pattern in (001) films, or wires periodic along y or cubic dots. 

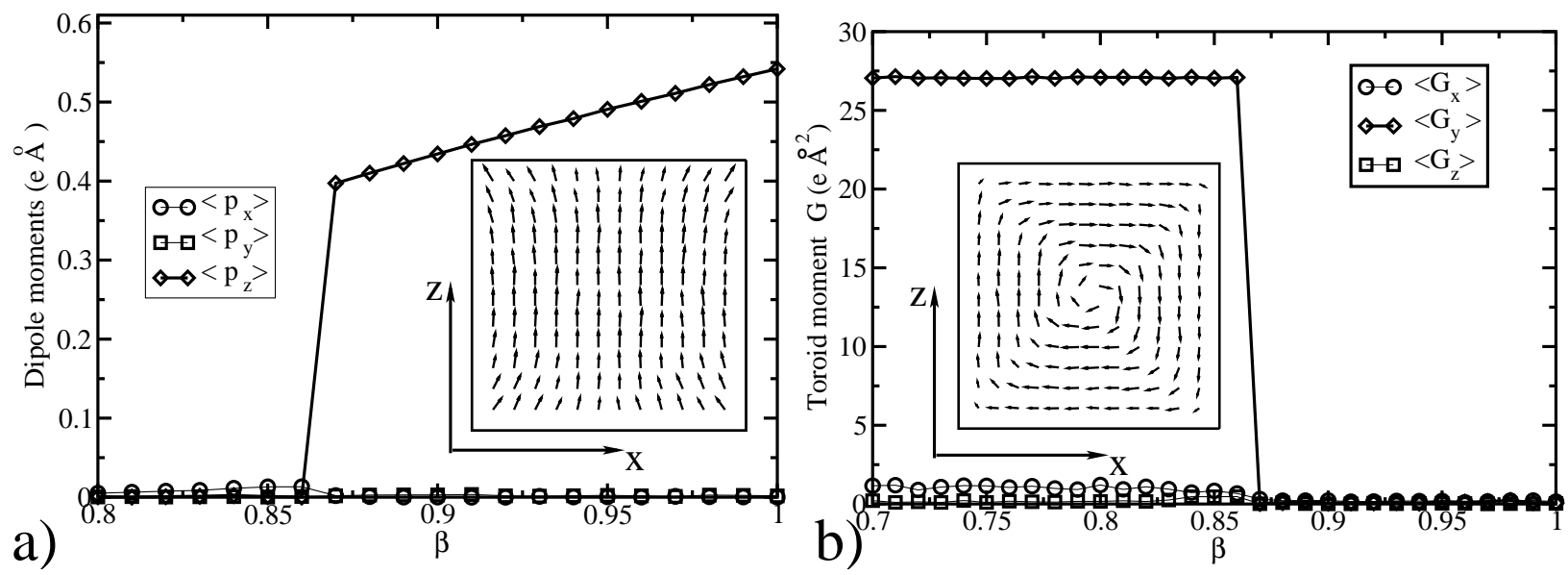

FIG. 3: a) Dipole moments and b) toroid moment of polarization $G$ in a PZT nanodot as a function of the screening coefficient $\beta$. Insets of the parts a) and b) show the polarization pattern for $\beta=1$ (SC conditions) and $\beta=0$ (OC conditions), respectively. 